\title{
PROGRESS ON THE RF COUPLING COIL MODULE DESIGN FOR THE MICE CHANNEL
}

\author{
D. Li, M. A. Green, S. P Virostek, and M. S. Zisman, LBNL/AFRD Berkeley CA 94720, USA; \\ W. Lau, A. E. White, and S. Q. Yang, Oxford University, Oxford OX1-3RH, UK
}

\begin{abstract}
We describe the progress on the design of the RF coupling coil (RFCC) module for the international Muon Ionization Cooling Experiment (MICE) at Rutherford Appleton Laboratory (RAL) in the UK. The MICE cooling channel design consists of one SFOFO cell that is similar to that of the US Study-II of a neutrino factory. The MICE RFCC module comprises a superconducting solenoid, mounted around four normal conducting 201.25-MHz RF cavities. Each cavity has a pair of thin curved beryllium windows to close the conventional open beam irises, which allows for independent control of the phase in each cavity and for the RF power to be fed separately. The coil package that surrounds the RF cavities is mounted on a vacuum vessel. The RF vacuum is shared between the cavities and the vacuum vessel around the cavities such that there is no differential pressure on the thin beryllium windows. This paper discusses the design progress of the RFCC module and the fabrication progress of a prototype $201.25 \mathrm{-MHz}$ cavity.
\end{abstract}

\section{INTRODUCTION}

The development of a muon collider or a neutrino factory requires low emittance muon beams. A key to the production of the low emittance muon beams is muon ionization cooling. A demonstration of muon cooling is thus essential to the development of muon accelerators and storage rings [1]. The international Muon Ionization Cooling Experiment (MICE) will be a demonstration experiment for muon ionization cooling in a configuration that was studied for a cooling channel for a neutrino factory in the US Study-II [2].

The proposed MICE experiment will test cooling on a low intensity muon beam generated by a plunging target in the proton beam of the ISIS ring at the Rutherford Appleton Laboratory in the United Kingdom. Once the muon beam is produced, it will be conditioned to give a desired emittance. The initial muon emittance will be measured in the first detector module using five planes of scintillating fibers that are within a $4 \mathrm{~T}$ spectrometer solenoid magnet. The muon beam then enters the first absorber focus coil (AFC) module containing the low- $Z$ absorber surrounded by a superconducting focus coil [3]. The muon beam loses both longitudinal and transverse momentum in the absorber. The RF coupling coil (RFCC) module is used to restore the muons to their original longitudinal momentum with RF cavities. The muon beam then enters the second AFC module. The MICE cooling channel consists of the three AFC modules separated by two RFCC modules. Once the muon beam exits the MICE cooling channel, its emittance is measured again in a second detector module that is essentially identical to the first detector module.

\section{THE RFCC MODULE}

The RFCC module consists of four 201.25 MHz RF cavities that are immersed in a $2.5 \mathrm{~T}$ solenoidal magnetic field generated by a superconducting coupling magnet [4]. A three-dimensional view of an RFCC module for MICE is shown in Figure 1, where the superconducting coupling magnet is shown to surround the four 201.25 $\mathrm{MHz}$ cavities. In order to increase the acceleration gradient within the RF cavity for a given input RF power, the cavities have thin beryllium windows to terminate RF fields at the beam iris. The cavity consequently becomes a closed cavity where the phase can be adjusted independently and power can be fed separately.

Due to the short muon lifetime and the presence of a strong solenoidal magnetic field, the cavity must be high gradient normal conducting. The beryllium windows provide nearly transparent windows to muon beams, while giving perfect RF conducting boundaries to RF fields. Large, $420-\mathrm{mm}$ diameter, beryllium windows are necessary to accommodate large transverse emittance muon beams.

To prevent the thin beryllium windows from experiencing differential pressure, the space outside the cavities must be evacuated. The cavity vacuum and the external vacuum are interconnected. The external vacuum is separated from the coupling magnet cryostat vacuum in order to prevent contamination of the RF vacuum by volatiles from the magnet multi-layer insulation.

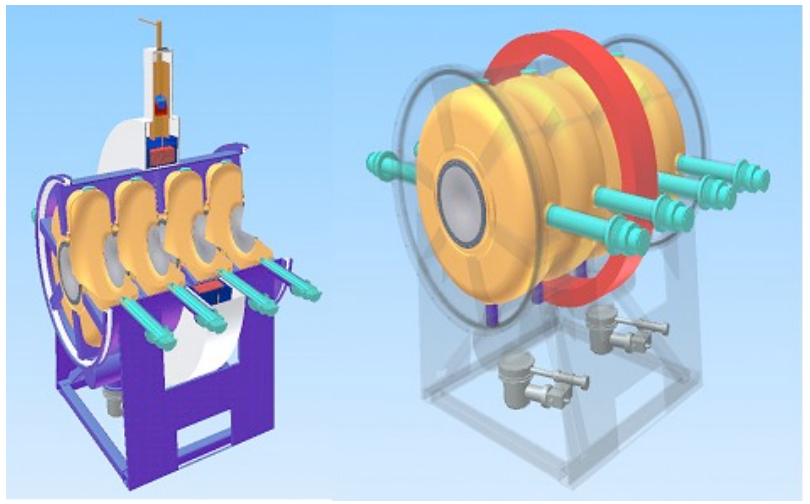

Figure 1: A three-dimensional view of the RFCC module for MICE

A cross-section view of the MICE cooling channel is shown in Figure 2. Three AFC modules and two RFCC modules are shown with their supporting structures and vacuum systems. 


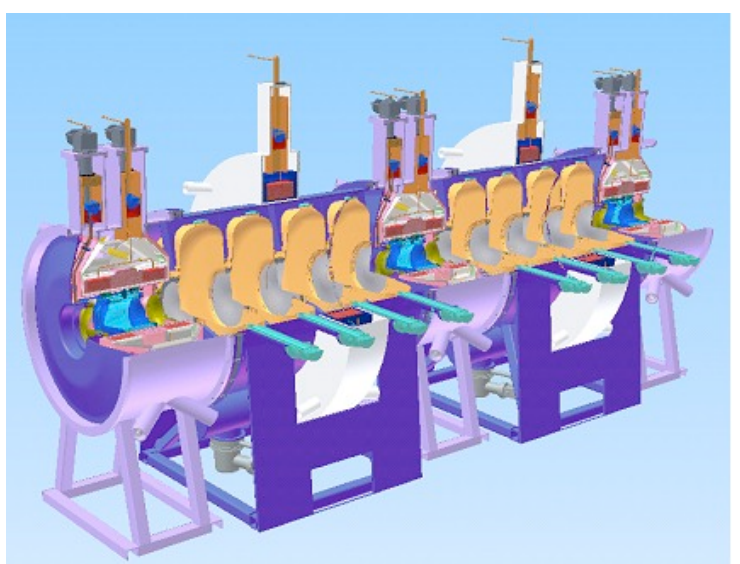

Figure 2: A cross section of the MICE cooling channel that includes three AFC modules and two RFCC modules.

\section{CAVITY DESIGN AND FABRICATION}

Figure 3 shows an exploded view of the $201.25 \mathrm{MHz}$ RF cavity. The cavity consists of a pair of spun copper shells. The shells are electron beam welded together to form the cavity. A stiffening ring is e-beam welded to each end of the cavity to strengthen the cavity shell for handling and vacuum, and to serve as a mounting point for tuners.

RF cavities for a muon cooling channel must be normal conducting and provide high accelerating gradient. The cavity design adopted a round, closed pillbox profile with a $420-\mathrm{mm}$ diameter beam iris to accommodate the large transverse emittance of muon beams. Curved beryllium windows are used to terminate RF fields at the beam iris. The cavity profile is optimized to minimize peak surface field and facilitate the fabrication process. The cavity therefore has high shunt impedance and gives a higher accelerating gradient for a given input RF power. The beryllium window diameter and thickness are determined by the muon beam profile at the cavity and the allowable temperature gradient and thermal stress within the window due to RF heating, respectively. The curvatures of the beryllium windows are oriented such that they point in the same direction to minimize frequency shift due to deformation of the windows under RF heating. The primary cavity parameters are listed in Table 1 .

As high as 4.6 MW peak power is needed to achieve 17 $\mathrm{MV} / \mathrm{m}$ accelerating gradient in the cavity. For such a high power, we choose to use two $\sim 4$ " coaxial loop RF couplers to power the cavity. The couplers were designed to be coupled at critical coupling, and were fabricated using standard off-the-shelf components. Spallation Neutron Source (SNS) type ceramic RF windows are incorporated into the coupler design. Figure 5 shows one of the loop couplers together with the SNS-type ceramic RF window. A water cooling line goes around the tip of the loop coupler to provide necessary cooling. The couplers have been fabricated and are being high power tested now at the SNS RF test lab.

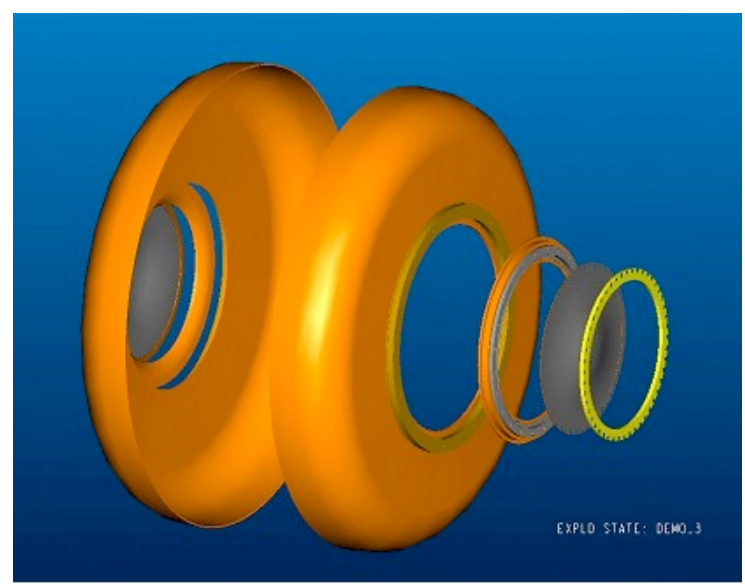

Figure 3: An exploded view of the MICE RF cavity design showing two spun copper shells, two thin curved beryllium windows and frames for holding the windows.

Table 1: Parameters for the 201.25 MHz MICE RF cavities

\begin{tabular}{lcc}
\hline Cavity length & 430 & $\mathrm{~mm}$ \\
Cavity inner radius & 610 & $\mathrm{~mm}$ \\
Cavity body thickness & 6 & $\mathrm{~mm}$ \\
No. of cavities per RFCC module & 4 & \\
Be window diameter & 420 & $\mathrm{~mm}$ \\
Be window thickness & 0.38 & $\mathrm{~mm}$ \\
Cavity shunt impedance & 22 & $\mathrm{M} \square / \mathrm{m}$ \\
Cavity quality factor $\mathrm{Q}_{0}$ & 53,000 & \\
Nominal accelerating gradient & 17.0 & $\mathrm{MV} / \mathrm{m}$ \\
Peak input RF power per cavity & 4.6 & $\mathrm{MW}$ \\
Ave power dissipation per cavity & 8.4 & $\mathrm{~kW}$ \\
Power dissipation on each window & 100 & $\mathrm{~W}$ \\
\hline
\end{tabular}

The first prototype cavity was e-beam welded together from two half shells spun from 6-mm thick copper sheets. There are four ports on the cavity. We have developed a technique to extrude a port through the e-beam welded equator joints. The technique has been successfully applied to the fabrication of the ports on the prototype cavity [5]. Figure 4 shows a photo of the prototype cavity during low power RF measurement conducted at Jefferson National Laboratory.

We have succeeded in the design and fabrication of curved thin beryllium windows. Thermal and structural analysis of the cavity with the beryllium windows using the ANSYS code is described in [6]. In comparison to the previously designed, fabricated and tested pre-stressed windows, the curved ones are thinner, yet mechanically strong and with predictable deflection. In addition, curved windows are significantly less expensive. Figure 6 shows a picture of a $0.38-\mathrm{mm}$ thick and $420-\mathrm{mm}$ diameter beryllium window made by the Brush-Wellman Company in California.

Progress on the coupling coil magnet design for the MICE cooling channel is reported in [7] at this conference and will not be repeated here. 


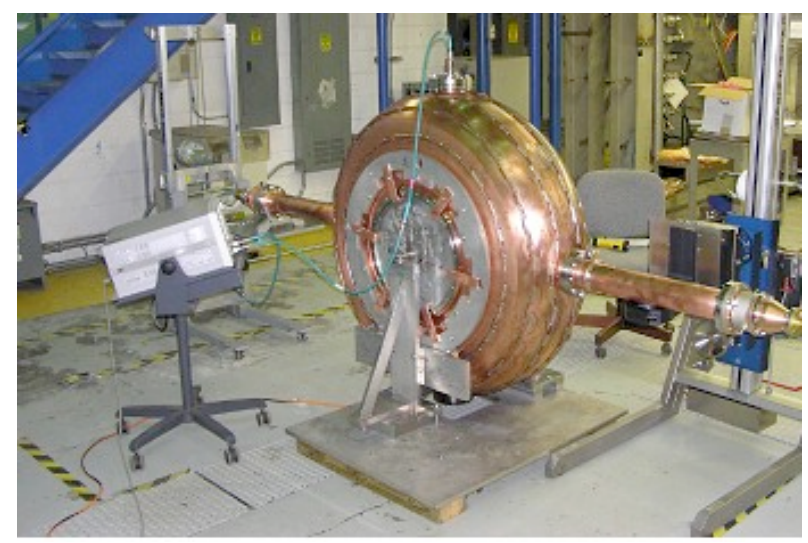

Figure 4: The 201.25 MHz prototype cavity with water cooling tubes (copper) welded onto the outer surface of the cavity. Two RF loop couplers were attached to the cavity for low power RF measurement of the frequency and coupling.

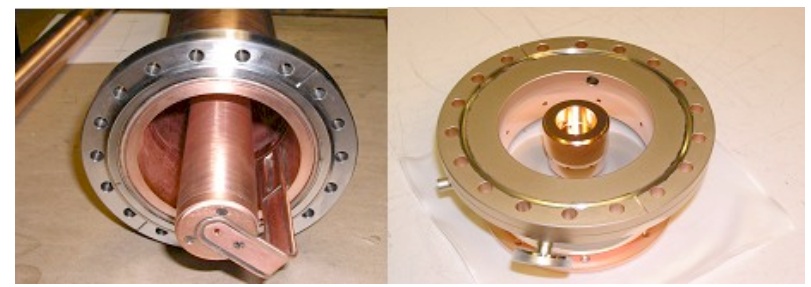

Figure 5: The coaxial loop coupler for the 201.25 prototype cavity (left), ceramic window (right) is an integral part of the coupler.

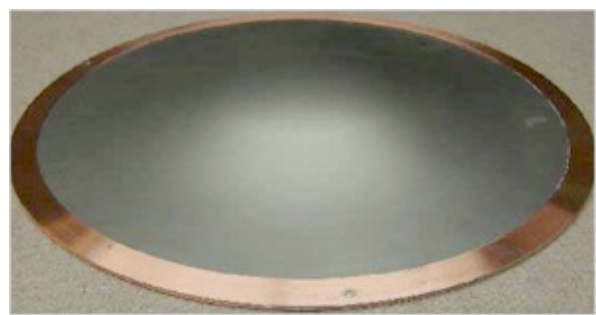

Figure 6: The first successful $0.38-\mathrm{mm}$ thick and $420-\mathrm{mm}$ diameter curved beryllium for the $201.25 \mathrm{MHz}$ cavity. The beryllium foil is sandwiched (brazed) between two annular copper frames.

\section{THE RFCC CAVITY VACUUM VESSEL}

The vacuum vessel for the RF cavity has been designed. A seal that connects the RFCC vacuum vessel to adjacent AFC modules has also been designed. The seal allows for the flexibility to remove and reinstall both AFC and RFCC modules from the MICE cooling channel, and permits longitudinal magnetic forces (up to $500-\mathrm{kN}$ ) to be carried from the AFC modules to the RFCC modules. The RFCC vacuum vessel is also designed to withstand the longitudinal magnetic forces generated by the coupling magnet in its various operating modes. The magnetic forces are then carried to the base plate of the experiment through the RFCC module stand. The vacuum within the RFCC vessel is totally isolated from the insulating vacuum within the coupling magnet vessel.

\section{CONCLUDING COMMENTS}

The RFCC module has been designed for MICE. The diameter of the coupling magnet is determined by the diameter of the 201.25 MHz RF cavities and the vacuum vessel that must go around the cavities. The length of the coupling magnet is determined by the space between the two RF couplers for center cavities and their tuners.

The baseline design of the MICE RF cavity is the same as that for an R\&D program of the US Neutrino Factory and Muon Collider Collaboration (MC). A prototype cavity has been fabricated and will be high power tested at the MUCOOL Test Area (MTA) at Fermilab this summer. The RFCC vacuum vessel has been designed. The design analysis includes the longitudinal magnetic forces that are transported to the vacuum vessel from the adjacent AFC modules and the coupling magnet.

\section{ACKNOWLEDGMENT}

This work was performed at the Lawrence Berkeley National Laboratory with the support of the Office of Science, United States Department of Energy under DOE contract number DE-AC03-76SF00098 and through the $\mathrm{MC}$.

\section{REFERENCES}

[1] R. B. Palmer, A. Sessler, A. Skrinsky, A. Tollestrup, et al., "Muon Colliders, “ Brookhaven National Laboratory Report BNL-62740, January 1996

[2] "MICE and International Muon Ionization Cooling Experiment Technical Reference Document,” ed. P. Drumm, (October 2004); http://www.isis.rl.ac.uk/accelerator/MICE/TR/MICE_Tech_ref.html

[3] S. Q. Yang, M. A. Green, G. Barr, et al., "The Mechanical and Thermal Design for the MICE Focusing Solenoid Magnet System," IEEE Transactions on Applied Superconductivity 15, No. 2 (2005)

[4] M. A. Green, S. Q. Yang, U. Bravar, et al., "The Mechanical and Thermal Design for the MICE Coupling Solenoid Magnet," IEEE Transactions on Applied Superconductivity 15, No. 2 (2005)

[5] R. Rimmer, S. Manning, R. Manus et al., "Fabrications of the Prototype Cavity for the Muon Ionization Cooling Experiment," Paper TPPT029 Proceedings of PAC-05 Conference, Knoxville TN, 16-20 May 2005

[6] S. Virostek, and D. Li "RF, Thermal, and Structural Analysis of the 201.25 MHz Muon Ionization Cooling Cavity," Paper TPPT029 Proceedings of PAC-05 Conference, Knoxville TN, 16-20 May 2005.

[7] M. Green, D. Li and S. Virostek, and et al., "Progress on the Coupling Coil For the MICE Channel", Paper MPPT059, PAC-05 Conference, Knoxville, TN, 16-20 May 2005. 